\title{
ANALYTIC CAPACITY, BILIPSCHITZ MAPS AND CANTOR SETS
}

\author{
John Garnett and JoAn Verdera
}

ABstract. We show that for planar Cantor sets analytic capacity is a bilipschitz invariant.

\section{Introduction.}

Let $E \subset \mathbb{C}$ be a compact plane set. The analytic capacity of $E$ is

$$
\gamma(E)=\sup \left\{\left|f^{\prime}(\infty)\right|: f \in A(E, 1)\right\}
$$

where

$$
A(E, 1)=\left\{f: f \text { is analytic on } \mathbb{C} \backslash E, f(\infty)=0 \text { and } \sup _{\mathbb{C} \backslash \mathbb{E}}|f(z)| \leq 1\right\}
$$

and $f^{\prime}(\infty)=\lim _{z \rightarrow \infty} z f(z)$. Then $\gamma(E)>0$ if and only if $A(E, 1)$ contains a non-constant function [G2]. A homeomorphism

$$
T: E \rightarrow T(E)
$$

is bilipschitz if $T$ and $T^{-1}$ satisify Lipschitz conditions

$$
\frac{1}{K}|z-w| \leq|T(z)-T(w)| \leq K|z-w|
$$

for all $z, w \in E$. This paper is concerned with the

Conjecture. If $T$ is bilipschitz, then

$$
\gamma(T(E)) \leq C(K) \gamma(E)
$$

where $C(K)$ depends only on the constant $K$ in (1.1).

Because $f \circ T$ and $f$ are seldom both analytic this conjecture may look foolhardy, but it has some supporting evidence. First, let $N(E)$ be the Newtonian capacity of $E$, which we define by

$$
N(E)=\sup \left\{\mu(E): \mu \text { Borel, } \mu>0, \sup _{z} \int_{E} \frac{d \mu(w)}{|z-w|} \leq 1\right\} .
$$

Then $\gamma(E) \geq N(E)$ because

$$
f(z)=\int_{E} \frac{d \mu(w)}{z-w} \in A(E, 1)
$$

Received June 14, 2002. 
for all $\mu$ in (1.2), and it is clear from the definition (1.1) that

$$
N(T(E)) \leq K N(E) .
$$

Second, suppose $E$ has finite one dimensional Hausdorff measure $\Lambda_{1}(E)<\infty$. Then by a deep theorem of David [D], $\gamma(E)=0$ if and only if $\Lambda_{1}(E \cap \Gamma)=0$ for every rectifiable curve $\Gamma$. Therefore,

$$
\gamma(T(E))=0 \text { if and only if } \gamma(E)=0
$$

when $\Lambda_{1}(E)<\infty$. If the rectifiable curve $\Gamma$ satisfies an Ahlfors condition:

$$
A^{-1} r \leq \Lambda_{1}(\Gamma \cap D(z, r)) \leq A r, z \in \Gamma, 0<r \leq \operatorname{diam}(\Gamma),
$$

then it is well known that for all $E \subset \Gamma$,

$$
C(A)^{-1} \Lambda_{1}(E) \leq \gamma(E) \leq C(A) \Lambda_{1}(E),
$$

and therefore

$$
\gamma(T(E)) \leq C(A, K) \gamma(E)
$$

because $T(\Gamma)$ is a rectifiable curve that also satisfies an Ahlfors condition. However, we do not have the preceding inequality with constant $C(K)$ independent of the curve $\Gamma$; indeed, that would be equivalent to the full conjecture.

Here we establish the conjecture for the Cantor sets with $\Lambda_{1}(E)=\infty$ that were studied in $[\mathrm{E}],[\mathrm{G} 2],[\mathrm{Ma}]$ and especially $[\mathrm{MTV}]$ and for their bilipschitz images. Let $E$ be a compact set of the form

$$
\begin{gathered}
E=\bigcap_{n=0}^{\infty} E_{n}, \\
E_{n}=\bigcup_{|J|=n} Q_{J}^{n},
\end{gathered}
$$

where $Q_{J}^{n}$ is closed, $J=\left(j_{1}, j_{2}, \ldots, j_{n}\right)$ is a multiindex of length $|J|=n$ with $j_{k} \in\{1,2,3,4\}$, and

$$
Q_{J, j_{n+1}}^{n+1} \subset Q_{J}^{n}
$$

for all $n$ and $J$. We assume there are constants

$$
0<a_{1}<a_{2}<1 / 2
$$

and

$$
c_{1}, c_{2}>0
$$

and a sequence $\sigma=\left(\sigma_{n}\right)$ such that $\sigma_{0}=1$ and

$$
\begin{gathered}
a_{1} \leq \frac{\sigma_{n+1}}{\sigma_{n}} \leq a_{2}, \\
\operatorname{diam}\left(Q_{J}^{n}\right) \leq c_{1} \sigma_{n}
\end{gathered}
$$

and

$$
\operatorname{dist}\left(Q_{J}^{n}, Q_{J^{\prime}}^{n}\right) \geq c_{2} \sigma_{n}, J \neq J^{\prime} .
$$


A paradigm for the set $E$ is obtained by letting $Q_{J}^{n}$ be a square of side $\sigma_{n}$ with sides parallel to the axes and requiring that $Q_{J, j}^{n+1}, j=1,2,3,4$, be the four corner subsquares of $Q_{J}^{n}$. In this case $E$ is the square Cantor set $E(\sigma)$ from [MTV], where it was proved that

$$
C^{-1}\left(\sum \frac{1}{4^{2 n} \sigma_{n}^{2}}\right)^{-1 / 2} \leq \gamma(E(\sigma)) \leq C\left(\sum \frac{1}{4^{2 n} \sigma_{n}^{2}}\right)^{-1 / 2}
$$

with constant $C$ independent of $\sigma$.

Now it is clear from (1.6) and (1.7) that if the sets $E$ and $E^{\prime}$ are defined by (1.3) and (1.4) for the same sequence $\left(\sigma_{n}\right)$, then

$$
T\left(E \cap Q_{J}^{n}(E)\right)=E^{\prime} \cap Q_{J}^{n}\left(E^{\prime}\right)
$$

defines a bilipschitz map from $E$ onto $E^{\prime}$ with constant $K=K\left(a_{1}, a_{2}, c_{1}, c_{2}\right)$. In particular, (1.3) - (1.7) describe all bilipschitz images of the Cantor set $E(\sigma)$.

Theorem. If $E$ is defined by (1.3),(1.4),(1.5),(1.6) and (1.7), then there is constant

$$
C=C\left(c_{1}, c_{2}, a_{1}, a_{2}\right)
$$

such that

$$
C^{-1}\left(\sum \frac{1}{4^{2 n} \sigma_{n}^{2}}\right)^{-1 / 2} \leq \gamma(E) \leq C\left(\sum \frac{1}{4^{2 n} \sigma_{n}^{2}}\right)^{-1 / 2}
$$

Corollary. There is a constant $C=C\left(K, a_{1}, a_{2}, c_{1}, c_{2}\right)$ such that

$$
C^{-1} \gamma(E) \leq \gamma(T(E)) \leq C \gamma(E)
$$

whenever $E$ is a Cantor set $E(\sigma)$ and $T$ is a bilipschitz map on $E$ satisfying (1.1) with constant $K$.

The Corollary follows immediately from the Theorem and the above discussion.

\section{Proof of Theorem}

The proof of the theorem depends on some exciting recent work of Tolsa [T1] and [T2]. Define the maximal function of a positive Borel measure $\mu$ as

$$
M_{\mu}(z)=\sup _{r} \frac{\mu(B(z, r))}{r}
$$

where $B(z, r)=\{w:|w-z|<r\}$. Let $R(z, w, \zeta)$ be the radius of the circle through $z, w$ and $\zeta \in \mathbb{C}$. Then $R(z, w, \zeta)^{-1}$ is called the Menger curvature of the triple $(z, w, \zeta)$. See Melnikov [Me]. Define the pointwise Menger curvature of $\mu$ at $z$ as

$$
c_{\mu}^{2}(z)=\iint \frac{1}{R(z, w, \zeta)^{2}} d \mu(w) d \mu(\zeta)
$$

and as in [V] define the Menger Potential of $\mu$ by

$$
U_{\mu}(z)=M_{\mu}(z)+c_{\mu}(z)
$$


Then the results we need from Tolsa [T1] and [T2] can be expressed as two inequalities:

$$
\gamma(E) \geq C_{1} \sup \left\{\mu(E): \sup _{z \in E} U_{\mu}(z) \leq 1\right\}
$$

and

$$
\gamma(E) \leq C_{2} \inf \left\{\mu(E): \inf _{z \in E} U_{\mu}(z) \geq 1\right\}
$$

with absolute constants $C_{1}$ and $C_{2}$. Let $E$ satisfy the hypothesis of the Theorem and define $\mu=\mu_{E}$ by

$$
\mu\left(Q_{J}^{n} \cap E\right)=4^{-n}
$$

Then for all $z \in E$

$$
M_{\mu}(z) \simeq \sup _{n} \frac{1}{4^{n} \sigma_{n}} .
$$

with constants depending only on $c_{1}$ and $c_{2}$. Note that $M_{\mu}(z)=\infty$ is possible for all $z \in E$.

The main difficulty in proving the Theorem comes from the obvious fact that a bilipschitz mapping may transform triples with positive Menger curvature into triples with zero curvature. For example the vertices of an equilateral triangle of side length 1 may be mapped into three collinear points. In the next example we will see that this may happen at all scales and locations, at least on a set of Hausdorff dimension less than 1.

Define a Cantor set as follows. Start with the interval $[0,1]$ and take 4 subintervals of length $1 / 5$ forming three equal gaps in $[0,1]$. Perform the same operation on each of these 4 intervals obtaining at the second step 16 intervals of length $1 / 25$. Proceeding inductively we obtain at the n-th step $4^{n}$ intervals $Q_{J}^{n}$ of length $5^{-n}$. Then (1.3) and (1.4) define a Cantor set $E$ associated to the sequence $\sigma_{n}=5^{-n}$. Define another Cantor set $E^{\prime}$ with the same sequence by starting with the unit square, taking 4 corner squares of side length $1 / 5$ at the first step and then proceeding inductively. As we pointed out before, there is a bilipschitz mapping $T$ from $E$ onto $E^{\prime}$ satisfying (1.8). Therefore the measure $\mu=\mu_{E}$ is transformed into the measure $\mu^{\prime}=\mu_{E^{\prime}}$. Notice that $c_{\mu}^{2}(z)=0, z \in E$, but $c_{\mu^{\prime}}^{2}(z)=\infty, z \in E^{\prime}$, as shown in [T1]. Nevertheless, it can be easily seen that $U_{\mu}(z)=\infty$ for all $z \in E$ and $U_{\mu^{\prime}}(z)=\infty$ for all $z \in E^{\prime}$.

Lemma 1. If E satisfies (1.3), (1.4), (1.5), (1.6) and (1.7), then

$$
c_{\mu}^{2}(z) \leq C\left(c_{1}, c_{2}\right) \sum_{n=1}^{\infty} \frac{1}{4^{2 n} \sigma_{n}^{2}} .
$$

Note that by (2.1), (2.3) and Lemma 1,

$$
\gamma(E) \geq C^{\prime}\left(c_{1}, c_{2}\right)\left(\sum \frac{1}{4^{2 n} \sigma_{n}^{2}}\right)^{-1 / 2}
$$

which gives the leftmost inequality in the Theorem. 
Proof of Lemma 1. The argument is from Mattila [Ma], and depends only on the trivial estimate

$$
\frac{1}{R(z, w, \zeta)} \leq \frac{2}{|z-w|}
$$

By symmetry we have

$$
c_{\mu}^{2}(z)=2 \iint_{|\zeta-z| \leq|w-z|} \frac{1}{R(z, w, \zeta)^{2}} d \mu(\zeta) d \mu(w) .
$$

Set

$$
A_{n}=\left\{(\zeta, w):|\zeta-z| \leq|w-z| \text { and } c_{1} \sigma_{n} \leq|w-z|<c_{1} \sigma_{n-1}\right\},
$$

for $n \geq 1$. Then clearly

$$
\begin{aligned}
& 2 \iint_{|\zeta-z| \leq|w-z|} \frac{1}{R(z, w, \zeta)^{2}} d \mu(\zeta) d \mu(w) \\
& \quad \leq C+\sum_{n=1}^{\infty} \iint_{A_{n}} \frac{8}{|w-z|^{2}} d \mu(\zeta) d \mu(w) \leq C \sum_{n=1}^{\infty} \frac{1}{4^{2 n} \sigma_{n}^{2}} .
\end{aligned}
$$

To prove the reverse inequality it is enough by (2.2) to show that

$$
U_{\mu}(z) \geq C\left(\sum \frac{1}{4^{2 n} \sigma_{n}^{2}}\right)^{\frac{1}{2}}
$$

for all $z \in E$.

Take $z \in E$. For each $n$ define $Q_{J}^{n}(z)$ as the $Q_{J}^{n}$ such that $z \in Q_{J}^{n}$ and following $[\mathrm{J}]$ define the Jones number

$$
\beta_{n}(z)=\inf \left\{\frac{\sup _{w \in E \cap Q_{J}^{n}(z)} \operatorname{dist}(w, L)}{\sigma_{n}}: L \text { is a line }\right\} .
$$

Thus $2 \beta_{n}(z) \sigma_{n}$ is the width of the narrowest strip containing $Q_{J}^{n}(z)$ and $\beta_{n}(z)$ is small if the inequality reverse to the trivial estimate (3.1) fails on $Q_{J}^{n}(z)$.

Lemma 2. Let $\delta=\frac{c_{2}}{2 \sqrt{2}}$. If

$$
\beta_{n}(z) \leq \delta \frac{\sigma_{n+p}}{\sigma_{n}}
$$

for some $p \geq 1$, then

$$
\sum_{k=1}^{p} 4^{n+k} \sigma_{n+k} \leq \frac{4 c_{1}}{c_{2}} 4^{n} \sigma_{n} .
$$

Proof of Lemma 2. By the definition of $\beta_{n}(z)$ there is a rectangle $R \supset Q_{J}^{n}(z)$ such that $Q_{J}^{n}(z)$ meets each of the four sides of $R$ and such that the smallest side of $R$ has length $2 \beta_{n}(z) \sigma_{n}$. Let $P$ denote the orthogonal projection onto the midline $L$ of $R$. By (1.7), the definition of $\delta$ and trigonometry we have for $j \neq k$

$$
\operatorname{dist}\left(P\left(Q_{J, j}^{n+1}\right), P\left(Q_{J, k}^{n+1}\right)\right) \geq \frac{c_{2}}{2} \sigma_{n+1} .
$$


Then because $R \cap L$ is connected,

$$
R \cap L \backslash \bigcup_{j=1}^{4} P\left(Q_{J, j}^{n+1}\right)
$$

contains three intervals each having endpoints in two distinct $P\left(Q_{J, j}^{n+1}\right)$ and each having length at least $\frac{c_{2}}{2} \sigma_{n+1}$.

Similarly, for $k=1,2, \ldots, p$ and for each $Q_{K}^{n+k-1} \subset Q_{J}^{n}(z), R \cap L$ contains three intervals having endpoints in two distinct $P\left(Q_{K, j}^{n+k}\right)$ and having length at least $\frac{c_{2}}{2} \sigma_{n+k}$. Since there are $4^{k-1}$ distinct $Q_{K}^{n+k-1} \subset Q_{J}^{n}(z)$, we obtain at least $3 \cdot 4^{k-1}$ pairwise disjoint intervals of length at least $\frac{c_{2}}{2} \sigma_{n+k}$ and furthermore, for $k>j$ these intervals are disjoint from the $3 \cdot 4^{j-1}$ intervals having endpoints in distinct $P\left(Q_{K^{\prime}}^{n+j}\right)$. The sum of the lengths of all these intervals is not larger than $\sqrt{2} \operatorname{diam}\left(Q_{J}^{n}(z)\right) \leq \sqrt{2} c_{1} \sigma_{n}$. Thus (3.4) follows.

Set

and for each positive integer $p$

$$
a_{n}=\frac{1}{4^{2 n} \sigma_{n}^{2}}
$$

$$
S=S(p)=\left\{n: 2 a_{n} \geq \operatorname{Max}_{1 \leq j \leq p} a_{n+j}\right\} .
$$

We need the following reformulation of Lemma 2.

Lemma 3. There exist a large positive integer $p=p\left(c_{1}, c_{2}\right)$ and a small positive number $\eta=\eta\left(a_{1}, p\right)$ such that if $n \in S(p)$ then

$$
\beta_{n}(z) \geq \eta \text {. }
$$

Proof of Lemma 3. If $\beta_{n}(z) \leq \delta \frac{\sigma_{n+p}}{\sigma_{n}}$ and $n \in S(p)$, then by Lemma 2

$$
\frac{1}{\sqrt{2}} p 4^{n} \sigma_{n} \leq \sum_{k=1}^{p} 4^{n+k} \sigma_{n+k} \leq \frac{4 c_{1}}{c_{2}} 4^{n} \sigma_{n},
$$

which gives an upper bound on $p$. If $p$ is chosen to be larger than $\sqrt{2} \frac{4 c_{1}}{c_{2}}$, then

$$
\beta_{n}(z) \geq \delta \frac{\sigma_{n+p}}{\sigma_{n}} \geq \delta a_{1}^{p} \equiv \eta
$$

whenever $n \in S(p)$.

The next lemma gives a relation between $\beta_{n}(z)$ and $c_{\mu}^{2}(z)$. See $[\mathrm{P}]$ for further results of this type. Assume from now on that $p$ and $\eta$ are given by Lemma 3 .

Lemma 4. If $\beta_{n}(z) \geq \eta$, then

$$
\iint_{F_{n}} \frac{1}{R(z, w, \zeta)^{2}} d \mu(w) d \mu(\zeta) \geq \frac{\epsilon_{0}}{4^{2 n} \sigma_{n}^{2}},
$$

where

$$
F_{n}=F_{n}(z)=\left\{\left(w_{1}, w_{2}\right) \in Q_{J}^{n}(z):\left|w_{j}-z\right| \geq \frac{\eta}{8} \sigma_{n}, j=1,2\right\}
$$


and $\epsilon_{0}$ is a positive constant depending on $\eta$.

Proof of Lemma 4. Take a point $b_{1}$ in $E \cap Q_{J}^{n}(z)$ such that $\left|b_{1}-z\right| \geq c_{2} \sigma_{n+1}$. By (1.5) and the definition of $\eta$ we then have $\left|b_{1}-z\right| \geq \eta \sigma_{n}$. Let $L$ be the line through $z$ and $b_{1}$. Since $\beta_{n}(z) \geq \eta$ there is a point $b_{2} \in E \cap Q_{J}^{n}(z)$ such that the distance from $b_{2}$ to $L$ is larger than $\left(\frac{\eta}{2}\right) \sigma_{n}$. Let $B_{j}$ denote the disc centered at $b_{j}$ of radius $\left(\frac{\eta}{8}\right) \sigma_{n}$. It is then clear that for some positive number $\epsilon_{1}$ depending on $\eta$ we have

$$
\mu\left(B_{j}\right) \geq \frac{\epsilon_{1}}{4^{n}}, j=1,2,
$$

and

$$
R\left(z, w_{1}, w_{2}\right) \leq \epsilon_{1}^{-1} \sigma_{n}, w_{j} \in B_{j}, j=1,2
$$

Thus

$$
\iint_{B_{1} \times B_{2}} \frac{1}{R(z, w, \zeta)^{2}} d \mu(w) d \mu(\zeta) \geq \frac{\epsilon_{1}^{4}}{4^{2 n} \sigma_{n}^{2}},
$$

which proves the lemma.

The next lemma shows that if $\sum a_{n}<\infty$ then $n \in S=S(p)$ for many values of $n$. Recall that $a_{n}=\frac{1}{4^{2 n} \sigma_{n}^{2}}$.

Lemma 5. We have

$$
\sum_{n=1}^{\infty} a_{n} \leq 2 p \sum_{n \in S} a_{n}+p M
$$

where $M=\sup _{n} a_{n}$.

Proof of Lemma 5. Set

$$
b_{n}=\max \left\{a_{j}:(p-1) n<j \leq p n\right\}, n=1,2, \cdots
$$

Let $N$ be a large integer and let $q$ be the positive integer such that $(p-1) q<$ $N \leq p q$. Denote by $G$ the set of integers $n$ such that $1 \leq n \leq q$ and $2 b_{n} \geq b_{n+1}$. Notice that an index $n \in G$ is good, in the sense that $b_{n}=a_{m}$ for some $m \in S$. Let $B$ stand for the set of indexes between 1 and $q$ which are not in $G$. Since

$$
\sum_{n \in B} b_{n} \leq \frac{1}{2} \sum_{n=0}^{q} b_{n+1},
$$

we have

$$
\sum_{n \in G} b_{n} \geq \frac{1}{2} \sum_{n=1}^{q} b_{n}-\frac{1}{2} b_{q+1}
$$

Therefore

$$
\sum_{n=1}^{N} a_{n} \leq p \sum_{n=1}^{q} b_{n} \leq 2 p \sum_{n \in G} b_{n}+p b_{q+1} \leq 2 p \sum_{n \in S} a_{n}+p M,
$$

and the lemma follows by sending $N \rightarrow \infty$. 
We can now complete easily the proof of (3.2). Since the domains of integration $F_{n}$ in Lemma 4 have bounded overlap, we get

$$
c_{\mu}^{2}(z) \geq \frac{\epsilon_{0}}{C} \sum_{n \in S} \frac{1}{4^{2 n} \sigma_{n}^{2}}
$$

where $C$ is some constant larger than 1 . By Lemma 5 and $(2.3)$ we then have, with another constant $C$,

$$
U_{\mu}^{2}(z) \geq \frac{\epsilon_{0}}{C}\left(\sum_{n \in S} \frac{1}{4^{2 n} \sigma_{n}^{2}}+M\right) \geq \frac{\epsilon_{0}}{C} \sum_{n=1}^{\infty} \frac{1}{4^{2 n} \sigma_{n}^{2}},
$$

which is $(3.2)$.

\section{Acknowledgements}

J. Garnett was supported in part by NSF Grant DMS-0070782. J. Verdera was supported in part by Grants 2001-SGR-00431, BFM2000-0361 and a fellowship from "Programa de Movilidad, MECD".

\section{References}

[D] G. David, Unrectifiable 1-sets have vanishing analytic capacity, Rev. Mat. Iberoamericana 14 (1998), 369-479.

[E] V. Ya. Eiderman, Hausdorff measure and capacity associated with Cauchy potentials, Math. Notes 63 (1998), 813-822.

[G1] J. Garnett, Positive length but zero analytic capacity, Proc. Amer. Math. Soc. 24 (1970), 696-699.

[G2] _ Analytic capacity and measure, Lecture Notes in Mathematics, Vol. 297. Springer-Verlag, Berlin-New York, 1972.

[J] P. W. Jones, Square functions, Cauchy integrals, analytic capacity, and harmonic measure, Harmonic analysis and partial differential equations (El Escorial, 1987), 24-68, Lecture Notes in Math., 1384, Springer, Berlin, 1989.

[Ma] P. Mattila, On the analytic capacity and curvature of some Cantor sets with non- $\sigma$ finite length, Publ. Math. 40 (1996), 195-204.

[Me] M.S. Melnikov, Analytic capacity: a discrete approach and the curvature of measure, Sb. Math. 186 (1995), 827-846.

[MTV] J. Mateu, X. Tolsa, J. Verdera, The planar Cantor sets of zero analytic capacity and the local T(b)-theorem, J. Amer. Math. Soc. 16 (2003), 19-28.

[P] H. Pajot, Notes on analytic capacity, rectifiability, Menger curvature and the Cauchy operator, to appear in Springer Lecture Notes.

[T1] X. Tolsa, On the analytic capacity $\gamma_{+}$, Indiana Univ. Math. J. 51 (2002), 317-343.

[T2] , Painleve's problem and the semiadditivity of analytic capacity, to appear in Acta Math.

[V] J. Verdera, On the T(1)-theorem for the Cauchy integral, Ark. Mat. 38 (2000), 183-199.

Department of Mathematics, UCLA, Los Angeles, CA 90095, U.S.A.

E-mail address: jbg@math.ucla.edu

Department de Matemàtiques, Universitat Autònoma de Barcelona, 08193 Bellaterra (Barcelona), Spain.

E-mail address: jvm@mat.uab.es 